\title{
Comportamiento agronómico y composición nutricional de diez variedades de pastos mejorados
}

\author{
Agronomic behavior and nutritional composition of \\ ten varieties of improved pastures \\ Eldin López Inga ${ }^{1}$; Manuel Oliva Cruz²; Pablo Huerta Fernández*3; \\ Rafael Urrelo Guerra ${ }^{4}$; Víctor Vásquez Arce ${ }^{5}$; Marco Honorio Acosta 6
}

\section{RESUMEN}

Esta investigación tuvo como objetivo evaluar el comportamiento agronómico y la composición nutricional de diez variedades de pastos mejorados, en las condiciones agroclimáticas del anexo de Nuevo Olmal en el distrito de Sonche. Para ello se utilizó el diseño experimental de bloques completos al azar (DBCA) con 10 tratamientos y 3 bloques. Las especies forrajeras gramíneas estudiadas fueron Gramilla, Raigrás ecotipo Cajamarquino, Raigrás tetraploide bianual Austral, Raigrás híbrido Boxer, Raigrás tetraploide Magnum, Festulolium, Dactylis glomerata Mammoth, Raigrás tetraploide anual Jumbo, Festuca Alta y Raigrás bianual tetraploide Hércules. Las variables evaluadas fueron rendimiento de forraje verde por hectárea, porcentaje de materia seca y contenido nutricional. Los resultados del análisis de varianza indicaron diferencias significativas $(\mathrm{p}<0.05)$ para todas las variables. La prueba de Bonferroni al 5\% determina que el mayor rendimiento logrado pertenece a Raigrás bianual tetraploide Hércules (26,63 t/ha), el mejor porcentaje de materia seca se encontró con D.G. Mammoth (20,21\%) y Festuca arundinaceae (19.39\%), la mayor cantidad de proteína la presentó el pasto local $(25,52 \%)$, el mayor porcentaje de fibra cruda se obtuvo con D.G. Mammoth $(17,68 \%)$ y pasto local $(17,28 \%)$, y el mayor valor relativo de digestibilidad in vitro de materia seca se halló en T. Magnum $(75,48 \%)$.

Palabras clave: pastos, gramíneas, Raigrás, composición.

\begin{abstract}
The main aim of this work was to evaluate the agronomic behavior and nutritional composition of ten varieties of improved pastures in the agroclimatic conditions of the Nuevo Olmal Annex in the Sonche district. For this, the experimental design of randomized complete blocks (DBCA) with 10 treatments and 3 blocks was used. The gramineous forage species studied were: Gramilla, ryegrass ecotype Cajamarquino, ryegrass tetraploid biannual Austral, ryegrass Hybrid Boxer, ryegrass tetraploid Magnum, Festulolium, Dactylis Glomerata Mammoth, ryegrass tetraploid annual Jumbo, Festuca Alta and ryegrass bi-annual tetraplioid Hercules. In our study, the variables studied were green forage yield per hectare, percentage of dry matter and nutritional content; the results of the analysis of variance indicated significant differences, $(p<0.05)$ for all the variables. The Bonferroni test at $5 \%$ determined that the highest yield achieved belongs to Ryegrass Biannual Tetraploide Hercules (26.63t/ha), the best percentage of dry matter was found with DG Mammoth (20.21\%) and Festuca arundinaceae (19.39\%), the highest amount of protein presents the local grass (25.52\%), the highest percentage of crude fiber was found with D.G. Mammoth (17.68\%) and local grass (17.28\%), the highest relative value of in vitro digestibility of dry matter was found in $\mathrm{T}$. Magnum (75.48\%).
\end{abstract}

Keywords: pastures, grasses, Ryegrass, composition.

\section{Introducción}

Según el Instituto Nacional de Estadística e Informática - INEI (2013), la superficie no agrícola del país está cubierta por pastos naturales que ocupan cerca de 18.1 millones de hectáreas (57\% de la superficie). Asimismo el estudio indica que en la región de la Sierra se encuentra el $60 \%$ de la

\footnotetext{
AGRORURAL, Ministerio de Agricultura y Riego. Chachapoyas, Amazonas, Perú.

Universidad Nacional Toribio Rodríguez de Mendoza. Amazonas, Amazonas, Perú.

Universidad Nacional Agraria La Molina. Lima, Perú.

Universidad Privada San Juan Bautista. Chorrillos, Lima, Perú.

Universidad Nacional de Cajamarca. Cajamarca, Perú.

6 Universidad Privada del Norte. Trujillo, La Libertad, Perú.

* Autor por correspondencia: apablohuerta@gmail.com
}

Fecha de Recepción: 28 de septiembre, 2020.

Fecha de Aceptación: 4 de febrero, 2021. 
superficie no agrícola del país y esta representa el $73,2 \%$ de la ganadería nacional, pero se reconoce que en gran parte son praderas degradadas, con tendencia a la retrogresión.

Uno de los obstáculos al momento de instalar pasturas de forma intensiva en las zonas altas de la región Amazonas son los suelos de baja cobertura de vegetación natural, que presentan limitantes nutricionales como nitrógeno, fósforo y elevado contenido de aluminio propio de zonas altas con frecuente presencia de precipitaciones. Asimismo, existen problemas de propiedades físicas como la infiltración y el anegamiento en estaciones lluviosas.

El distrito de Sonche, Amazonas, Perú, abarca 11,326 hectáreas de superficie total, de las cuales un 6,18\% es área reforestada con la especie Pinus patula y en gran porcentaje tierras aptas para silvopastura. La gran mayoría son pastos naturales con predominio de la especie Stipa ichu o paja. Mayormente son praderas naturales que carecen de un manejo adecuado, y además no compensan los requerimientos nutricionales del ganado bovino, lo cual está relacionado con el nivel de degradación y baja fertilidad de los suelos.

Los pastos son el componente fundamental para la alimentación del ganado en la dehesa. Florísticamente son muy variados y abundan especies herbáceas anuales, como gramíneas, con reducida presencia de perennes (Olea, Verdasco y Paredes, 2011). Asimismo, las leguminosas son fuente importante de proteínas y proporcionan mayor estabilidad en la producción de forraje. Gracias a su resistencia a la sequía mantienen su valor nutritivo durante la estación seca. En los sistemas de silvopastoreo intensivos, la mejor alternativa para la producción de ganado con un sistema sostenible es a través de la utilización de leguminosas asociadas con gramíneas (UNAM, 2017).

Los pastos y forrajes juegan un papel muy importante desde el punto de vista agrícola, por cuanto muchos pastos se desarrollan en suelos donde otros cultivos no se pueden producir ni biológica ni económicamente. Cabe señalar que los pastos ayudan a conservar el suelo, ya que por su cubierta densa y protectora evitan o reducen la erosión, al impedir el contacto directo de las gotas de lluvia sobre el terreno descubierto, y al disminuir la velocidad y el poder de arrastre del agua superficial o de escorrentía en suelos de pendientes, lo que favorece una mayor infiltración, absorción y abastecimiento de agua. Por consiguiente, los pastos constituyen una de las mejores alternativas para ser incluidos en la rotación de cultivos y para la protección de cuencas hidrográficas (Sierra, 2005).

Esta investigación se realizó con el objetivo de evaluar el comportamiento productivo de diez variedades de pastos mejorados, su adaptación y productividad en las condiciones del área geográfica de estudio. Ello motivado por la necesidad de ganar área productiva e incluir la producción sostenible, así como recopilar una buena información sobre la variedad con mejor rendimiento y productividad al momento de instalar pasturas a nivel intensivo, y de esta manera contribuir con el desarrollo económico del ganadero de la zona, de la región y del país.

\section{Material y métodos}

La investigación se realizó en el anexo de Nuevo Olmal, distrito de Sonche, provincia, Chachapoyas, región Amazonas, Perú, entre los meses de octubre del 2017 y abril del 2018.

\section{Localidad: Anexo - Nuevo Olmal}

Las muestras fueron 10 variedades de pastos y forrajes de clima frío con diversas características agronómicas, cultivadas bajo las condiciones del anexo Nuevo Olmal, distrito Sonche, provincia Chachapoyas, Amazonas (Tabla 1).

\section{Técnicas}

Altura de planta (AP): se realizó el muestreo de forma manual de las muestras señaladas para la evaluación. Se midió la altura en centímetros desde el cuello de la planta hasta el punto más alto sin modificar su contextura.

Número de macollos (NM): el número de macollos se evaluó de forma manual en cada muestra al momento del corte.

Forraje verde (FV): los cortes se realizaron en el mismo lugar de cada unidad experimental para evitar pérdidas por deshidratación. Se cortó y se pesó con el apoyo de una balanza analítica. Los resultados fueron extrapolados a una hectárea expresada en $\mathrm{kg}$ de $\mathrm{FV} / \mathrm{ha}$.

Materia seca (MS): se recolectaron $100 \mathrm{~g}$ de forraje verde como muestra de cada tratamiento. Posteriormente fue transportado al laboratorio de nutrición animal y bromatología de alimentos de la UNTRM, acondicionado en trozos de 2-3 cm y 
etiquetados adecuadamente. Se llevaron a la estufa a una temperatura de $105^{\circ} \mathrm{C}$, por un periodo de 24 horas. Para el cálculo de la materia seca se empleó la siguiente fórmula:

$$
\% \mathrm{MS}=\frac{p f}{p i} \times 100
$$

Donde:

$\%$ MS: porcentaje de materia seca; $P f:$ peso final (g) y pi: peso inicial (g)

Análisis bromatológico: para identificar esta variable se realizó un muestreo de las 10 plantas en evaluación extrayendo una determinada cantidad de cada una, para un total de $100 \mathrm{~g}$. Luego se trasladó la muestra y se colocó en una estufa a $60^{\circ} \mathrm{C}$ por un tiempo de 72 horas, procediendo a molerla. Después de triturada se puso en reposo para recuperar un porcentaje de humedad. Posteriormente se determinó el contenido nutricional utilizando la técnica de espectroscopia de Infrarrojo Cercano (NIRs) con el equipo NIRs del Laboratorio de Bromatología y Nutrición Animal de la Universidad Nacional Toribio Rodríguez de Mendoza.

\section{Procedimiento}

Se ubicó una parcela dentro del área comunal del anexo Nuevo Olmal. Para su selección se tomó en cuenta el acceso y la ligera pendiente del terreno.
El área total destinada para el estudio fue de $332,8 \mathrm{~m}^{2}$. Cada unidad experimental tuvo las siguientes dimensiones: largo: 3,0 m; ancho: 2,0 m, con callejones de $0,40 \mathrm{~m}$ entre tratamientos y calles de ingreso de 1,0 m entre bloques. Dentro de cada unidad experimental estuvieron distribuidas las plantas de pastos a una densidad de $0,02 \times 0,02 \mathrm{~m}$ entre plantas y surcos (Figura 1 y 2 ).

Para conocer las condiciones de fertilidad del suelo se tomó una muestra de forma representativa, y se trasladó al Laboratorio de Suelos y Agua de la Universidad Nacional Toribio Rodríguez de Mendoza de Amazonas. Se recolectó la muestra previa a la instalación.

Con el objetivo de identificar la especie que predomina en el área de estudio se recopilaron muestras de las gramíneas. Nombre común reportado: "Gramilla"; lugar de colecta: área geográfica del estudio. Luego se llevaron las muestras al Instituto de Investigación para el Desarrollo Sustentable de Ceja de Selva INDES-CES para su identificación, Una vez revisadas las características morfológicas del ejemplar, se determinó que la muestra botánica correspondía a la especie Digitaria sanguinalis (L.) Scop., perteneciente a la familia de las Poáceas, identificada en el estudio como variedad uno (V1).

Se realizaron las labores de labranza con anticipación. Esta actividad se hizo con la tracción de un motocultor, por tres pases en diferentes tiempos. En el segundo pase se aplicó la enmienda cálcica según cálculos. Las especies forrajeras fueron elegidas por sus características agronómicas

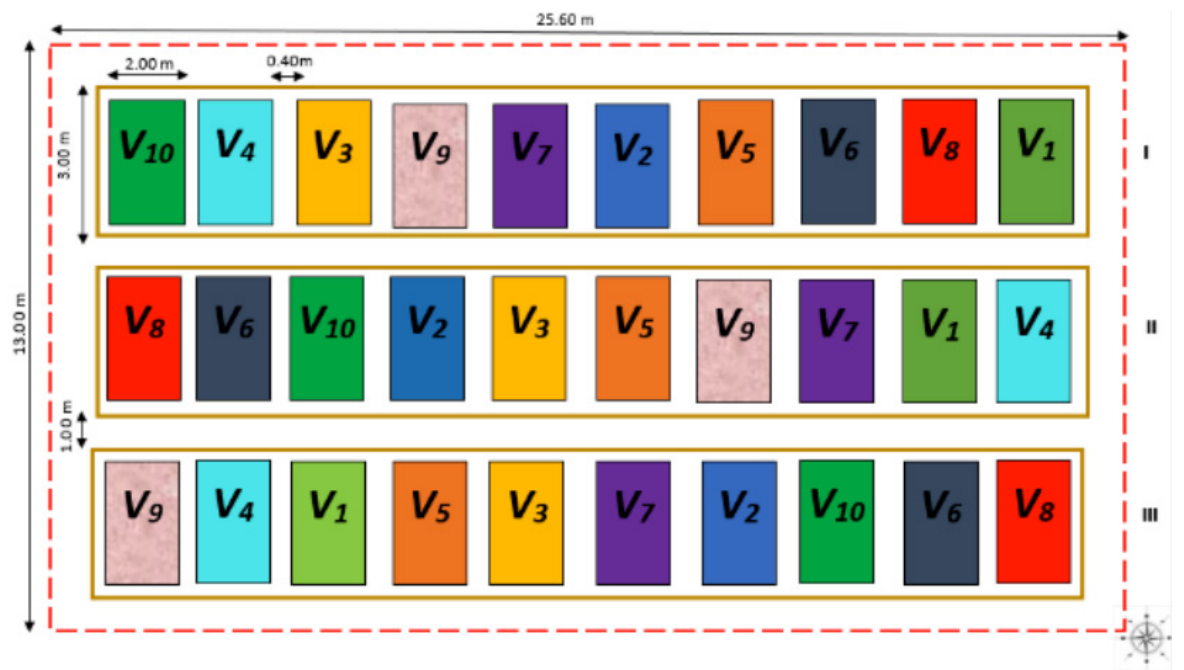

Figura 1. Croquis de la distribución de parcelas en campo experimental. 


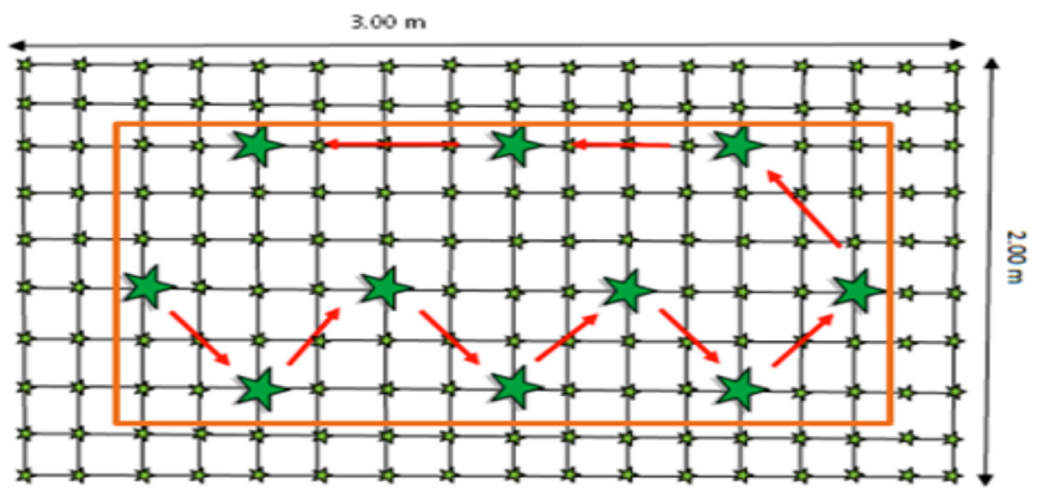

Figura 2. Detalle de la unidad experimental.

descritas en la ficha técnica y entre ellas se tomó en cuenta el rendimiento, valor nutritivo, altitud y tolerancia a acidez del suelo. Se seleccionaron nueve variedades comerciales conformadas por raigrases, festuca, Dactylis, adicionando la especie Digitaria sanguinalis (L.) Scop identificada. Se utilizaron como fertilizantes orgánicos guano de las islas y roca fosfórica. La fórmula de fertilización NPK para instalación fue 96-237-22 y la de mantenimiento después de cada corte 50-45-10. En lugares con presencia de humedad debido a la alta pluviosidad las pérdidas de $\mathrm{N}$ son altas y llega a desperdiciarse un $60 \%$ del $\mathrm{N}$ aplicado. Para evitar esto se fraccionaron las aplicaciones.

La siembra se realizó colocando la semilla al golpe. Se sembró un aproximado de 4 semillas, las cuales una vez emergidas fueron seleccionadas extrayendo las plantas con defectos y dejando una sola planta por golpe. Se evaluaron tres cortes: primer corte 90 días después de instalado; segundo corte 165 días después de instalado, y tercer corte 240 días después de instalado.

\section{Resultados}

En la Tabla 2 se observan los resultados del análisis de varianza para el peso del forraje verde, los cuales indican que existen diferencias
Tabla 1. Variedades de pastos incluidos en la investigación.

\begin{tabular}{ll}
\hline & \multicolumn{1}{c}{ Variedad } \\
\hline $\mathrm{V}_{1}$ & Gramilla. \\
$\mathrm{V}_{2}$ & Raigrás ecotipo Cajamarquino. \\
$\mathrm{V}_{3}$ & Raigrás tetraploide bianual Austral. \\
$\mathrm{V}_{4}$ & Raigrass híbrido Boxer. \\
$\mathrm{V}_{5}$ & Raigrás tetraploide Magnum. \\
$\mathrm{V}_{6}$ & Festulolium. \\
$\mathrm{V}_{7}$ & Dactylis glomerata Mammoth. \\
$\mathrm{V}_{8}$ & Raigrás tetraploide anual Jumbo. \\
$\mathrm{V}_{9}$ & Festuca arundinaceae (alta). \\
$\mathrm{V}_{10}$ & Raigrás bianual tetraploide Hercules. \\
\hline
\end{tabular}

significativas para la fuente variedad, dado que el valor de significación ( $p$-valor $=0.000$ ) es menor al 0,05. Este resultado muestra que las variedades de pasto en estudio se diferencian unas de otras por el peso del forraje verde.

Al realizar la prueba de Bonferroni (Tabla 3), se evidencia que los resultados de las variedades $\mathrm{V}_{6}, \mathrm{~V}_{2}, \mathrm{~V}_{3}, \mathrm{~V}_{5}, \mathrm{~V}_{8} \mathrm{y} \mathrm{V}_{10}$, cuyos pesos frescos del forraje oscilan entre $17029,7 \mathrm{~kg} \mathrm{~V}_{6}$ y $26638,6 \mathrm{~kg}$ $\mathrm{V}_{10}$, son estadísticamente iguales y superiores a los de las variedades $\mathrm{V}_{9}, \mathrm{~V}_{7} \mathrm{y} \mathrm{V}_{1}$, cuyos pesos frescos del forraje son $7780,3 \mathrm{~kg}, 7045,8 \mathrm{~kg}$ y $1846,7 \mathrm{~kg}$.

En la Tabla 4 se observan los resultados del análisis de varianza para el peso seco, los cuales

Tabla 2. Análisis de varianza para el rendimiento.

\begin{tabular}{lcccc}
\hline Fuente de variación & Grados de libertad & Cuadrados medios & F calculado & p-valor \\
\hline Bloques & 2 & 280011202 & 8.02 & 0.003 \\
Tratamientos & 9 & 576752461 & 16.53 & 0.000 \\
Total & 89 & & & \\
\hline
\end{tabular}


Tabla 3. Prueba de significación de Bonferroni para el rendimiento.

\begin{tabular}{rccccccc}
\hline Variedades & Rendimiento (Tn) & \multicolumn{5}{c}{ Agrupación } \\
\hline 10 & 26,638 & A & & & & \\
8 & 23,252 & A & B & & & \\
5 & 21,950 & A & B & & & \\
3 & 19,700 & A & B & & & \\
2 & 18,528 & A & B & C & & \\
6 & 17,029 & A & B & C & D & \\
4 & 13,316 & & B & C & D & \\
9 & 7,780 & & & C & D & E \\
7 & 7,045 & & & & D & E \\
1 & 1,846 & & & & & E \\
\hline
\end{tabular}

Tabla 4. Análisis de varianza para el peso seco.

\begin{tabular}{lcccrr}
\hline Fuente de variación & Grados de libertad & Suma de cuadrados & Cuadrados medios & F calculado & p-valor \\
\hline Bloques & 2 & 1,169 & 0,5843 & 0,27 & 0,767 \\
Tratamientos & 9 & 453,509 & 50,3899 & 23,29 & 0,000 \\
\hline
\end{tabular}

indican que existen diferencias significativas para la fuente tratamientos, dado que el valor de significación (p-valor $=0.000$ ) es menor al 0,05. Este resultado demuestra que las variedades de pasto en estudio se diferencian unas de otras por el peso seco.

Al aplicar la prueba de Bonferroni (Tabla 5), se observa que las variedades $\mathrm{V}_{7} \mathrm{y} \mathrm{V}_{9}$, cuyos pesos secos son 20,21 y $19,39 \%$, respectivamente, son estadísticamente iguales y superiores al resto; es decir, que los pesos secos de estas variedades se diferencian del peso seco del resto de variedades. Las variedades $\mathrm{V}_{1}, \mathrm{~V}_{2}, \mathrm{~V}_{8}, \mathrm{~V}_{6}, \mathrm{~V}_{10}, \mathrm{~V}_{4} \mathrm{y} \mathrm{V}_{3}$, cuyos pesos secos oscilan entre $13,66 \%$ para la $\mathrm{V}_{3}$ y $15,75 \%$ para la $\mathrm{V}_{1}$, presentan resultados estadísticamente iguales y son inferiores a los dos primeros.

La prueba de Bonferroni para el porcentaje de proteína (ver Tabla 6) arrojó que la variedad $\mathrm{V}_{1}$ es estadísticamente igual al porcentaje de proteínas de las variedades $\mathrm{V}_{9}, \mathrm{~V}_{4}, \mathrm{~V}_{7}, \mathrm{~V}_{8}, \mathrm{~V}_{6}, \mathrm{~V}_{2} \mathrm{y} \mathrm{V}_{5}$. El resultado del $\mathrm{V}_{1}(25,52 \%)$ se diferencia de los resultados de las variedades $\mathrm{V} 3 \mathrm{y} \mathrm{V}_{10}$, que son 18,54 y $17,90 \%$, respectivamente.

En la Tabla 7 se observa que las variedades $\mathrm{V}_{7} \mathrm{y} \mathrm{V}_{1}$, cuyos porcentajes de fibra cruda son de $17,68 \%$ y $17,28 \%$, respectivamente, resultan estadísticamente iguales. El resultado de la $\mathrm{V}_{7}$ es diferente al del resto de variedades en estudio. Por otro lado, la variedad $\mathrm{V}_{9}$, cuyo resultado fue $11,15 \%$, es estadísticamente menor al resto.
Tabla 5. Prueba de significación de Bonferroni para el peso seco.

\begin{tabular}{ccccc}
\hline Variedades & Peso seco $(\%)$ & \multicolumn{3}{c}{ Agrupación } \\
\hline 7 & 20,21 & A & & \\
9 & 19,39 & A & & \\
1 & 15,75 & & B & \\
2 & 15,01 & & B & C \\
8 & 14,23 & & B & C \\
6 & 14,10 & & B & C \\
10 & 13,77 & & B & C \\
4 & 13,70 & & B & C \\
3 & 13,66 & & B & C \\
5 & 12,59 & & & C \\
\hline
\end{tabular}

Tabla 6. Prueba de Bonferronipara el porcentaje de proteína.

\begin{tabular}{ccll}
\hline Variedades & Proteína $(\%)$ & \multicolumn{2}{c}{ Agrupación } \\
\hline 1 & 25,52 & $\mathrm{~A}$ & \\
9 & 23,00 & $\mathrm{~A}$ & $\mathrm{~B}$ \\
4 & 22,78 & $\mathrm{~A}$ & $\mathrm{~B}$ \\
7 & 20,17 & $\mathrm{~A}$ & $\mathrm{~B}$ \\
8 & 19,38 & $\mathrm{~A}$ & $\mathrm{~B}$ \\
6 & 19,09 & $\mathrm{~A}$ & $\mathrm{~B}$ \\
2 & 19,07 & $\mathrm{~A}$ & $\mathrm{~B}$ \\
5 & 18,88 & $\mathrm{~A}$ & $\mathrm{~B}$ \\
3 & 18,54 & & $\mathrm{~B}$ \\
10 & 17,90 & & $\mathrm{~B}$ \\
\hline
\end{tabular}

Se muestra en la Figura 3 que las alturas de las variedades $\mathrm{V}_{2}, \mathrm{~V}_{10}, \mathrm{~V}_{8}, \mathrm{~V}_{5}, \mathrm{~V}_{3} \mathrm{y} \mathrm{V}_{6}$ oscilan 
Tabla 7. Prueba de significación de Bonferroni para el porcentaje de fibra cruda.

\begin{tabular}{cccccccc}
\hline Variedad & Fibra cruda (\%) & \multicolumn{5}{c}{ Agrupación } \\
\hline 7 & 17,68 & A & & & & \\
1 & 17,28 & A & B & & & \\
2 & 15,68 & & B & C & & \\
5 & 14,33 & & & C & D & \\
3 & 13,63 & & & & D & \\
10 & 13,49 & & & & D & \\
8 & 13,04 & & & & D & E \\
4 & 12,78 & & & & D & E \\
6 & 12,61 & & & & D & E \\
9 & 11,15 & & & & & E \\
\hline
\end{tabular}

entre $25.04 \mathrm{~cm}$ para la $\mathrm{V}_{6}$ y $29,87 \mathrm{~cm}$ para la $\mathrm{V}_{2}$, son estadísticamente iguales y se diferencian de la $\mathrm{V}_{4} \mathrm{y} \mathrm{V}_{7}$. Por otro lado, la variedad $\mathrm{V}_{1}$, cuya altura de planta es de $11,41 \mathrm{~cm}$, resulta estadísticamente diferente e inferior al resto.

En la Tabla 8 se observa que las variedades $\mathrm{V}_{10}, \mathrm{~V}_{6}, \mathrm{~V}_{2}, \mathrm{~V}_{5}, \mathrm{~V}_{4}, \mathrm{~V}_{8}$ y $\mathrm{V}_{3}$, cuyo número de macollos oscila entre 39 para la $V_{3}$ y 51 para la $\mathrm{V}_{10}$, son estadísticamente iguales. Por otro lado, los resultados obtenidos con las variedades $\mathrm{V}_{9}, \mathrm{~V}_{7}$ y $\mathrm{V}_{1}$ cuyo número de macollos es de 29, 24 y 11, respectivamente, son diferentes a los 7 primeros.

Según la Figura 4, se observa en el biplot realizado que la variedad Ryegrass bianual tetraploide Hércules tiene mejores resultados en todas las variables evaluadas, mientras que la variedad Gramilla obtuvo bajos valores en todas las variables evaluadas. Además, se muestra que las variables de composición nutricional (fibra cruda, peso seco y proteína) con las demás variables se comportan de manera independiente.

\section{Discusión}

Vásquez, Quilcate y Oliva (2017) realizaron investigaciones sobre rendimiento de Ryegrass, donde obtuvieron rendimientos entre 12 y $15 \mathrm{t} / \mathrm{ha}$. Estos resultados son menores a los encontrados en este trabajo. Los pesos frescos de las variedades en estudio oscilaron entre $1,84 \mathrm{t} / \mathrm{ha}$ (pasto local) y 26,63 t/ha (Raigrás bianual tetraploide Hércules), rendimientos que distan de los anteriores. Probablemente el mayor rendimiento logrado en nuestro trabajo se deba a las condiciones de suelo de la localidad y a las buenas prácticas de abonamiento, tal como lo requieren los pastos
Tabla 8. Prueba de significación de Bonferroni para el número de macollos.

\begin{tabular}{cccccc}
\hline Variedades & Número de macollos & \multicolumn{5}{c}{ Agrupación } \\
\hline 10 & 51 & A & & & \\
6 & 51 & A & & & \\
2 & 49 & A & & & \\
5 & 46 & A & & & \\
4 & 44 & A & & & \\
8 & 44 & A & & & \\
3 & 39 & A & B & & \\
9 & 29 & & B & C & \\
7 & 24 & & & C & D \\
1 & 11 & & & & D \\
\hline
\end{tabular}

mejorados tipo Raigrás, para la producción de forraje verde.

Según la Figura 3, se comprueba que no existe diferencia significativa entre las variedades estudiadas en lo que respecta a la altura; sin embargo, se ha logrado formar cuatro subgrupos mediante la prueba de comparaciones múltiples. En el primer grupo que presenta una mayor altura está la especie Lolium (variedades 2, 10, 8, 5, 3 y 6 ), mientras que de las diez variedades estudiadas encontramos que la Gramilla es la especie que tiene un menor crecimiento.

En cuanto al número de macollos, se observa la formación de cuatro subconjuntos en los que las variedades que conforman cada uno no tienen unadiferencia significativa. En cuanto al rendimiento expresado en toneladas como aparece en la Tabla 3, los rendimientos obtenidos con las variedades registradas mensualmente con una

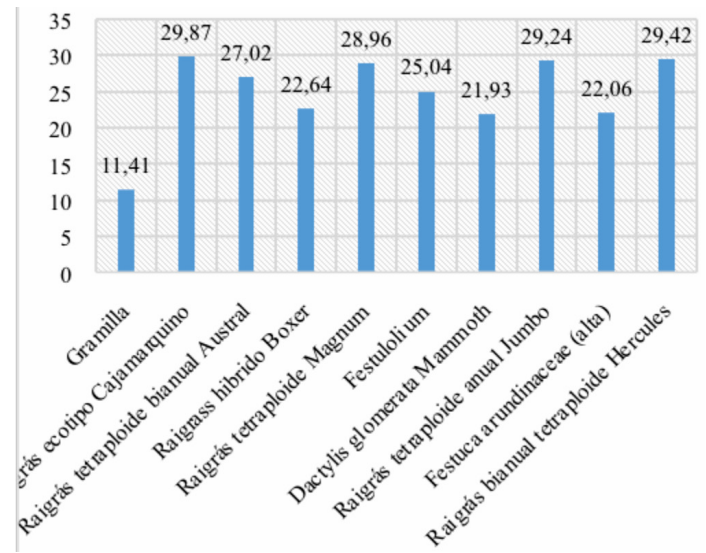

Figura 3. Altura de las variedades en centímetros. 
proyección de un año concuerdan con los estudios de Cadena, García y Castro (2019), quienes en genotipos de Raigrás consiguieron resultados en intervalos de 20,76 a 33,36 t/ha/año.

En relación con el contenido de proteínas, como se registra en la Tabla 6, los datos de las variedades $\mathrm{V} 3$ y $\mathrm{V}_{10}$ con $18,54 \%$ y $17,90 \%$, respectivamente, son muy aproximados a los resultados obtenidos por Posada et al. (2013) en Ryegrass híbrido tetraploide $(18,8 \%)$ y Ryegrass anual tetraploide $(18,9 \%)$.

En la Figura 4, a través de un análisis multivariado se estableció que la variedad que presenta los mejores resultados de los indicadores estudiados es Raigrás bianual tetraploide Hércules. Si bien la Figura 4 comparaba porcentualmente las características de cada variedad evaluada, el análisis multivariado evidenció la contribución de varios factores (indicadores) para determinar la mejor variedad.

\section{Conclusiones}

Los mayores rendimientos del peso fresco oscilaron entre 17 t/ha (Festulolium) y 26,6 t/ ha (Raigrás bianual tetraploide Hércules), y los menores rendimientos se encontraron con las variedades Festuca arundinaceae (7,78 t/ha), Dactylis glomerata Mammoth $(70,45 \mathrm{t} / \mathrm{ha}) \mathrm{y}$ Gramilla o pasto local (1,84 t/ha). El pasto local fue el que obtuvo el menor rendimiento en el peso fresco, y se diferencia de los pastos mejorados evaluados en esta investigación. Con respecto a la materia seca, los pastos que lograron mayores rendimientos son Dactylis glomerata Mammoth $(20,21 \%)$ y Festuca arundinaceae $(19,39 \%)$. El pasto local y el resto de pastos evaluados obtuvieron resultados similares que oscilaron entre 12,59\% y $15,75 \%$. Estos se diferencian significativamente de los dos primeros.

Con respecto a las proteínas, los mayores rendimientos oscilaron entre $18,88 \%$ (Raigrás tetraploide Magnum) y 25,52\% (pasto local). El porcentaje de proteína más bajo se encontró con las variedades Raigrás bianual tetraploide Hércules $(17,90 \%)$ y con V3 $(18,54 \%)$.

El pasto local fue el que obtuvo mayor contenido de proteínas que los pastos mejorados evaluados en esta investigación. Con respecto a
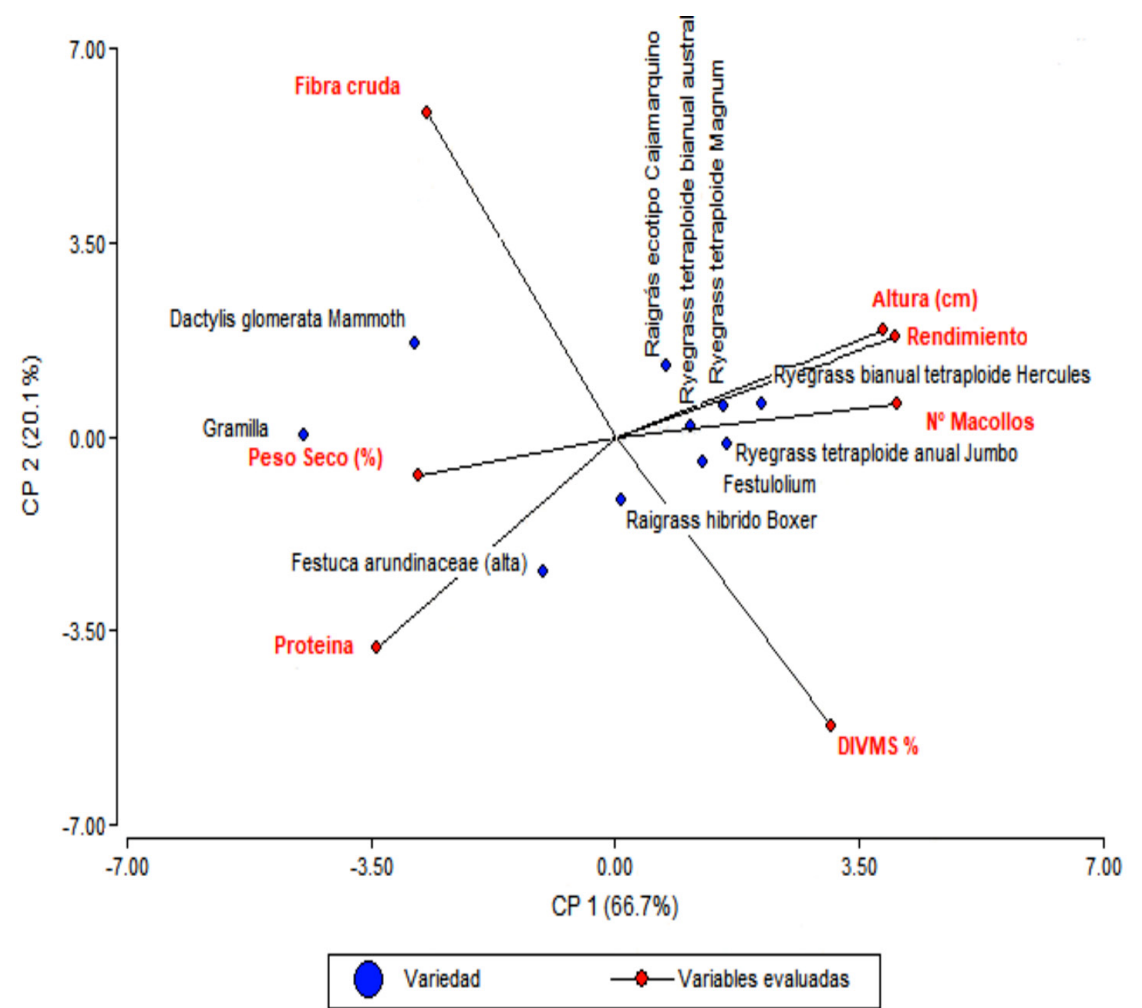

Figura 4. Análisis multivariantes de las variedades estudiadas. 
la fibra cruda, los pastos que lograron mayores rendimientos fueron Dactylis glomerata Mammoth y pasto local, cuyos porcentajes son $17,68 \%$ y $17,28 \%$, respectivamente. El menor porcentaje se encontró con Festuca arundinaceae, cuyo promedio fue de $11,15 \%$. Con respecto al pasto local, los contenidos de fibra cruda de los pastos mejorados fueron menores. Por último, se determinó mediante un análisis multivariado que la variedad que presenta mejores características de las evaluadas en este trabajo es Raigrás bianual tetraploide Hércules.

\section{Literatura Citada}

Cadena, M.M; García, M.A; Castro; E.

2019. Estabilidad fenotípica de genotipos de Lolium sp. en el trópico alto de Nariño, Colombia. Revista de Agronomía Mesoamericana, Universidad de Costa Rica, 30(2): 483-495.

Universidad Nacional Autónoma de México.

2017. Características nutrimentales de gramíneas, leguminosas y algunas arbóreas forrajeras del trópico mexicano: Fracciones de proteína (A, B1, B2 y C), carbohidratos y digestibilidad in vitro. $1^{\mathrm{a}}$. ed. Departamento de Nutrición y Bioquímica, UNAM. México. 172 p.

Instituto Nacional de Estadística e Informática (INEI)).

2013. Resultados Definitivos. IV Censo Nacional Agropecuario 2012. INEI. Lima, Perú. Disponible en: http://www.minagri.gob.pe/portal/especial-iv-cenagro/320libros-electronicos Consultado: 3/jun/2020.
Olea, L.; Verdasco, M.; Paredes, J.

2011. Características y producción de los pastos de las dehesas del S.O. de la Península Ibérica. Pastos, 20(21): 131-156.

Posada, S; Cerón, J.M; Arena, J.; Hamedt, J.F.; Álvarez, A.

2013. Evaluación del establecimiento de ryegrass (Lolium sp.) en potreros de kikuyo (Pennisetum clandestinum) usando la metodología de cero labranza. Universidad CES Medellín, Colombia. Revista CES Medicina Veterinaria y Zootecnia, 8(1): 23-32.

Sierra, J.O.

2005. Fundamentos para el establecimiento de pasturas y cultivos forrajeros (Segunda). Universidad de Antioquía. Medellín, Colombia. 262 p.

Vásquez, H.; Quilcate, C.; Oliva, M.

2017. Evaluación de quince variedades de gramíneas forrajeras para el mejoramiento alimenticio del ganado bovino en la cuenca ganadera Florida. Revista de Investigación en Ciencia y Biotecnología Animal, 1(1): 69-75. 